\title{
Educación política en Argentina. Participación juvenil, política educativa, enseñanza y contextos*
}

\author{
María-Inés Jorquera** \\ Universidad Nacional de Avellaneda, Argentina \\ Laura Radetich $* * *$ \\ Universidad de Buenos Aires, Argentina \\ https://doi.org/10.15446/frdcp.n20.90974
}

\section{Resumen}

El presente artículo tiene como propósito interpretar las transiciones de la educación política en Argentina haciendo foco en el cambio socio histórico a través de la dinámica de la participación política juvenil, los cambios en las políticas educativas y las políticas en torno a adolescentes y jóvenes, junto a las transformaciones de los contextos

\footnotetext{
* Artículo recibido: 30 de septiembre de 2020 / Aceptado: 8 de febrero de 2021 / Modificado: 17 de marzo de 2021. El presente artículo original es el resultado del proyecto de investigación y vinculación intercátedras de Didáctica Especial de la Historia y Prácticas de la Enseñanza en la Universidad de Buenos Aires (Argentina) Facultad de Filosofía y Letras y Didáctica de la Enseñanza de la Historia en el Nivel Superior en la Universidad Nacional de Avellaneda (UNDAV) - Departamento de Humanidades y Artes en el marco de la Convocatoria 2016/2019 PRIICA + 11643626 - UNDAV. Los proyectos PRIICA UNDAV no cuentan con financiamiento.

* Magíster en Ciencias Sociales con orientación en Educación por la Flacso, Argentina. Profesora de la Universidad Nacional de Avellaneda y de la Universidad de Buenos Aires (Argentina) - Facultad de Filosofía y Letras. Correo electrónico: marinejorquera77@gmail.com (iD https://orcid.org/0000-0002-4567-7934

***** Magíster en Nuevas Tecnologías de la Educación por la Universidad de Salamanca, España. Profesora de la Universidad de Buenos Aires (Argentina) - Facultad de Filosofía y Letras. Correo electrónico: radetich2020@gmail.com (iD) https://orcid.org/0000-0001-6289-460X

Cómo citar
}

Jorquera, M. I. y Radetich, L. (2021). Educación política en Argentina. Participación juvenil, política educativa, enseñanza y contextos. FORUM. Revista Departamento Ciencia Política, 20, 36-61. https://doi.org/10.15446/frdcp.n20.90974 
sociopolíticos. Para tal fin, se desarrolló un abordaje interpretativo documental sobre legislación educativa, diseños curriculares, planes de estudio, revistas educativas, discursos y textos escolares históricos y actuales. El análisis se centrará en los fines de la formación ciudadana junto a los enfoques y prácticas de enseñanza expresados en las reformas curriculares y de los planes de estudio y sus contextos, con el propósito de comprender el camino histórico que concluyó en el actual modelo de educación política basada en el ejercicio político pleno por parte de la juventud.

Palabras clave: educación política; políticas educativas; planes de estudios; cultura ciudadana; participación política juvenil.

\title{
Political Education in Argentina. Youth Participation, Educational Policy, Teaching and Contexts
}

\begin{abstract}
The purpose of this article is to interpret the transitions of political education in Argentina, focusing on the socio-historical change through the dynamics of the political participation of youth, changes in educational policies and policies regarding adolescents and young people, together with the transformations of the sociopolitical contexts. For this purpose, a documentary interpretive approach was developed on educational legislation, curricular designs, study plans, educational magazines, speeches and historical and current school texts. The analysis will focus on the purposes of citizenship training together with the teaching approaches and practices expressed in the curricular and study plan reforms and their contexts, in order to understand the historical path that resulted in the current education model policy based on full political exercise by the youth.
\end{abstract}

Keywords: political education; educational policies; curricula; citizen culture; youth political participation.

\section{Introducción}

Hacia 1884, como parte de un vasto conjunto de políticas fundacionales para la naciente República Argentina, tomaba cuerpo la Ley de Educación Común 1420 que regulaba la educación primaria bajo los principios de obligatoriedad, gratuidad y gradualidad. Dicha Ley, reconocida hasta hoy como la norma fundante del sistema educativo argentino, sentó 
las bases de un consistente proyecto nacional de enfoque patriótico y con propósitos civilizatorios en pos de la inserción del país al concierto internacional de naciones. En 1905, como complemento de la misma, se establece la Ley 4874, conocida como Ley Láinez, con el propósito de extender la educación pública al creciente territorio nacional y a la educación de los hijos de los inmigrantes que se sumaban al país como parte de la política de poblamiento que traía los brazos necesarios para el desarrollo de las producciones agrícola ganaderas centrales en el modelo económico primario exportador.

En los albores del sistema educativo argentino la educación patriótica era un mecanismo de adoctrinamiento propio de la escuela primaria. Escudé (1990) sostiene una hipótesis central en la que afirma que: "...la cultura política fue distorsionada de una manera contraria al progreso y al buen funcionamiento de las instituciones (liberales) por medio de un adoctrinamiento autoritario y dogmático, grandilocuentemente chauvinista" (p. XVIII).

Para describir cómo ese adoctrinamiento se materializó en la escuela a partir de 1908, Escudé utilizó como fuentes publicaciones educativas de distintas épocas, fundamentalmente El monitor de la educación y diversos manuales escolares. Este recorrido le permitió afirmar que se trató del único proyecto argentino que sobrevivió a diversos regímenes políticos y gobiernos de signo partidario contrapuestos. En el prólogo de su obra podemos leer:

[...] el origen de los mitos [de las pérdidas territoriales argentinas] era una cosa y su difusión hasta convertirse en un elemento generalizado de la cultura política argentina era otra muy distinta, y ya en ese ensayo anuncié mi intención de llevar a cabo un relevamiento de textos de enseñanza para verificar la medida en que elementos del nacionalismo territorial argentino habían sido difundidos por el sistema educativo. Este trabajo, titulado "Contenido nacionalista de los textos de geografía de la enseñanza primaria y secundaria en la Argentina, 1879-1986", fue publicado en mi Patología del Nacionalismo: el caso argentino... Es así como un interés por la historia de la política exterior argentina me condujo a un estudio de sus condicionantes culturales, de los mecanismos educativos específicos que habían coadyuvado a gestar y perpetuar esa cultura, y de las ideologías que habían inspirado a quienes así moldearon el sistema educativo de este país...Esta premisa implica, para las ciencias sociales todas, una revaloración de la historia de los contenidos educativos, que debería ubicarse en un lugar central en el estudio del comportamiento humano y del desarrollo político y económico. Por cierto, creo además que la historia de los contenidos educativos es central para la comprensión de la irracionalidad en los comportamientos políticos, tanto internos como internacionales. (Escudé, 1990, p. XVI)

En este marco de relevancia que Escudé le adjudica a la educación como productora de la trama cultural, política y material, cobra relevancia la interpretación del derrotero histórico de la educación política en Argentina, las políticas públicas educativas que la modelaron y sus efectos en la participación política juvenil hasta conformarse un modelo de educación política basada en el ejercicio político pleno por parte de la juventud. 


\section{Nacionalismo e instrucción cívica durante el periodo conservador 1900-1916}

La conceptualización del periodo como "conservador" nos permite iniciar la problematización en torno al carácter de la dimensión sociopolítica de Argentina a principios de siglo XX frente al carácter liberal del modo en que se encaró su desarrollo material bajo un modelo de crecimiento hacia afuera. Así entendidos los términos, Argentina en 1900 vivía una lucha ideológica entre liberalismo y autoritarismo, y la educación habría de ser uno de los campos de batalla más notables, donde se tejerán los atributos conservadores en materia social y política.

Ya hacia 1910, la postura autoritaria se había impuesto en los programas de educación patriótica y, aunque no se lo reconociera explícitamente, el espíritu liberal y desarrollista que había inspirado la Ley de Educación Común 1420 de 1884 -su principio de laicidad- quedó sepultado, imponiéndose, finalmente, un espíritu dogmático y autoritario. Es decir, liberal en lo económico y conservador en lo social y político pasaría a ser un lema para la dirigencia argentina próxima al centenario de la patria.

Incluso, tras la ampliación democrática a través del sufragio universal, secreto y obligatorio de la Ley 8871 de 1912 y el triunfo electoral a nivel nacional, en 1916, de la Unión Cívica Radical (UCR) - partido moderno de origen revolucionario y popular- derrotando a los conservadores, no se verificó ninguna modificación en la educación política, que continuó fundándose en el adoctrinamiento autoritario y dogmático, claramente chauvinista. Los programas continuaron inalterados y la orientación patriótica permaneció vigente en los planes y en la rutina. No obstante, cierta moderación y pluralismo se observan en la revista El monitor de la educación perteneciente al Consejo Nacional de Educación de la Nación Argentina, que apenas comienza a incorporar nuevas voces pero que continúa sosteniendo la perspectiva conservadora de hegemónica. Incluso, ante el crecimiento del socialismo y el anarquismo, se profundiza la persecución ideológica de la cual florecerán los ideales xenófobos tan caros al conservadurismo y sus leyes de control social.

A partir de 1920 se realiza un entrecruzamiento entre las materias historia e instrucción cívica que comenzaba con la revalorización de los símbolos - de la patria, de la bandera y del himno- y cuyo objetivo pedagógico era ir de la historia a la patria y de la patria a la historia. La instrucción cívica orientaba su enseñanza en ideas elementales de familia, sociedad, gobierno y fuerzas de seguridad. En la medida en que disminuían los contenidos patrióticos en los programas de historia, empezó a crecer esta tendencia en los de instrucción cívica. Estas dos materias terminaron complementando y reforzando una educación patriótica en el marco de la doctrina del tutelado de niños, niñas, adolescentes y jóvenes. 
Finalizando la Primera Guerra Mundial comenzó una nueva etapa de inmigración y de esta manera los intelectuales del periodo cuestionaban la cualidad de la integración de esos inmigrantes a la patria mientras sostenían a la educación como la herramienta eficaz para la homogenización social de los hijos de los nuevos pobladores.

En 1930, tras la crisis del modelo económico hacia afuera, la oligarquía ganadera gesta el primer golpe de Estado en la República Argentina para desplazar al gobierno de la UCR, tomando, también, las riendas de la educación argentina. Sin embargo, fue recién después del Congreso Eucarístico de Buenos Aires en octubre de 1934 que se marcó el punto de agonía de la Argentina liberal y el nacimiento de una Argentina nacionalista y católica. La oligarquía encontró la unidad detrás de los objetivos de la iglesia, adoptando al catolicismo como "la religión de nuestros mayores". El orden, la norma y la disciplina se constituirán en los pilares de la educación para la patria y la escuela, se erigirá como el baluarte de la construcción del nacionalismo -incluso frente a la institución familiar - frente al peligro de las ideas y las costumbres foráneas.

Los sucesivos gobiernos sostuvieron la misma perspectiva del nacionalismo católico para la educación nacional al punto de instaurar la enseñanza de la religión católica en todas las escuelas públicas del país mediante el decreto 18411 de 1943, que luego sería convertido en ley en el año 1946 durante el gobierno peronista.

Se puede advertir que durante el periodo de conformación del sistema educativo argentino hasta los albores del primer gobierno peronista, la educación política, tuvo como principales propósitos el adoctrinamiento nacionalista de carácter autoritario y dogmático, reiteradamente chauvinista, sobre adolescentes y jóvenes tutelados por el estado, la familia y la iglesia, pero donde la escuela obtuvo un papel primordial en formar el carácter patriótico. De tal modo, la escuela se constituirá en la institución que legitimará a la educación política como una cuestión de estado.

\section{La ampliación de la participación ciudadana y la cultura cívica durante el primer y segundo gobiernos peronistas (1946-1955)}

Desde la llegada al poder, el peronismo tuvo como objetivo orientar la educación política de los jóvenes en los valores de independencia económica, soberanía política y justicia social, a los cuales consideraba como basamentos para la enseñanza en el nivel medio. El Decreto 26944 de 1947 expresa aquellos objetivos de la siguiente forma: 
... formar la conciencia nacional de los alumnos, despertando y fomentando el amor a la patria y el sentido de la propia responsabilidad. Debe crear en el alma de los adolescentes una clara visión de nuestro papel en el mundo y la convicción de que les corresponde la empresa de lograr para la República una nueva etapa de sensorio espiritual y material. (citado en Cora y Rodríguez, 2015, p. 190)

En el marco de un modelo de crecimiento hacia adentro, el gobierno peronista amplió el ámbito de la participación ciudadana a través de la profundización de la educación ciudadana, ya no bajo los principios de la educación para el nacionalismo patriótico precedente, sino a través de la formación de la conciencia nacional basada en una doctrina nacional y popular estructurada en torno a la creciente movilización de los sectores populares, la organización de los adolescentes y jóvenes en la política nacional y en la distribución de bienes materiales y culturales.

A partir de 1952, las reformas educativas propuestas por el segundo Plan Quinquenal y los principios dogmáticos de la Reforma Constitucional de 1949 habían sido declaradas objetivo educativo fundamental. Entre todos los cambios, se incorporó en 1953 una nueva materia, cultura ciudadana en el Ciclo Básico del Nivel Medio, que según el Ministerio de Educación de la Nación se presenta como el "núcleo de irradiación y centro de atracción de toda la labor del aula" (Cora y Rodríguez, 2015, p. 191).

La introducción de contenidos para la nueva conciencia argentina serán impartidos haciendo algunas modificaciones en la materia historia y especialmente desde la nueva asignatura: cultura ciudadana. Este vínculo entre saberes históricos y saberes ciudadanos fue utilizado desde mediados del siglo XIX en Argentina, tal como lo vimos en la anterior etapa de conformación de la educación política a través del sistema escolar. Si bien cultura ciudadana era considerada el núcleo central del plan de estudios, se la consideraba un área transversal a toda la carga horaria y, tal como había ocurrido anteriormente, los contenidos eran también reforzados en el diseño curricular de historia y, por esta razón, solo ocupaba dos horas semanales a lo largo de la formación básica del nivel medio.

La modificación del plan de estudios y de los contenidos en el nivel medio se realizó cuando fue evidente que la educación política en la escuela primaria era consistente con la idea de nacionalismo que se esperaba de sus egresados mientras que en la formación de jóvenes la selección curricular contenía aún rasgos humanistas cosmopolitas. Asimismo, la expansión de la educación media de 48794 alumnos en 1943 a 97 306 en 1955 (Puiggrós, 2003), renovó las expectativas del gobierno nacional en torno a la educación política. En consecuencia, el proceso de masificación del nivel posibilitó la profundización de la educación política transformada, ahora, como formación de la conciencia nacional. 


\section{La formación política y las organizaciones estudiantiles durante el peronismo}

En 1953 el Ministro de Educación Armando Méndez San Martín creó la Unión de Estudiantes Secundarios (UES) para nuclear a los jóvenes en torno de la cuestión nacional y orientar su participación en la cosa pública. Ya en 1921 se había creado la Federación Juvenil Comunista (FJC), que se unió a partir de 1945 a la Federación Mundial de la Juventud Democrática. Más adelante, en 1955, con el fin de hacer frente a la UES y la FJC fue creada la Unión de Estudiantes Nacionalistas Secundarios (UENS), católicos nacionalistas pro nazis que actuaran como grupo de choque a partir de 1955 hasta 1962. Con la creación de dichas agrupaciones estudiantiles la participación política de los jóvenes se profundizaba a la vez que se creaban vertientes estudiantiles tensamente enfrentadas.

Los movimientos de estudiantes secundarios fueron la base de la juventud que luego luchará en el enfrentamiento de 1958 entre católicos y liberales por la educación "laica o libre”. Paradójicamente, para la prensa, el Jefe de Policía, el Ministro de Educación y el presidente radical desarrollista Arturo Frondizi (1958-1962), esa práctica confrontativa que movilizaba a las juventudes era consecuencia directa de la escasa formación cívica que los estudiantes habrían recibido en la década peronista y a su erosión de los principios de autoridad y disciplina. Para dichos actores la participación estudiantil no era observada como un proceso de democratización de la sociedad civil sino como una disrupción al orden político que el gobierno radical desarrollista buscaba instituir.

Este fenómeno participativo de los estudiantes continuó reiterándose a lo largo de la segunda parte del siglo XX, en donde los estudiantes secundarios volvieron, una y otra vez, a ser parte del debate político, incluso a fuerza de ser encarcelados, torturados y asesinados por reivindicar sus derechos.

\section{La cartera educativa nacional, su condición ministerial y la educación política: una cuestión de denominaciones y rangos}

Desde 1898 hasta 1949 el Ministerio en donde el Estado generaba las políticas educativas se llamaba Ministerio de Justicia e Instrucción Pública, o sea que la educación formaba parte de una cartera que estaba abocada a varias áreas de la función pública. Fue a partir del peronismo que esto cambió, dándole el nombre y la importancia que tenía la educación dentro del gobierno y lo nombró Ministerio de Educación por primera vez en la historia. Luego, los vaivenes de la política argentina hicieron que este ministerio, incluso, hasta perdiera su condición y se lo bajara al status de secretaria de gobierno. Finalmente, con el advenimiento de la democracia, desde 1983, el Ministerio de Educación fue revalorizado pudiendo mantener su rango. 
La materia dedicada a la educación política también tuvo diversas denominaciones a lo largo de la historia del sistema educativo y de la relevancia que la formación política poseía en cada contexto.

Tabla 1. Variación en la nominación de la materia para la enseñanza de la educación política desde 1953 al presente

\begin{tabular}{|l|l|}
\hline Año de creación & Nombre de la materia a lo largo del tiempo \\
\hline 2011 (continua) & Trabajo y ciudadanía \\
\hline 2011 (continua) & Política y ciudadanía \\
\hline 2010 (continua) & Salud y adolescencia \\
\hline 2007 (continua) & Construcción ciudadana \\
\hline 1993 & Formación ética y ciudadana \\
\hline 1984 & Educación cívica \\
\hline 1980 & Formación moral y cívica \\
\hline 1976 & Formación cívica \\
\hline 1973 & Estudio de la Realidad Social Argentina (ERSA) \\
\hline 1955 & Educación democrática \\
\hline 1953 & Cultura ciudadana \\
\hline
\end{tabular}

Fuente: elaboración propia.

Tal como se observa en la tabla 1, tras el golpe de Estado desatado contra el segundo gobierno peronista en 1955, se suprime la materia cultura ciudadana, vaciando la educación política como promotora de la movilización juvenil y la cuestión nacional y popular e instaurando la denominación educación democrática para la nueva versión de una educación política con objetivo disciplinador, retornando a la perspectiva de la instrucción cívica basada en la transmisión del sistema constitucional, los deberes y los derechos cívicos

De tal modo, las variaciones en torno al rango, cualidades y denominaciones de la cartera educativa y la materia dedicada a la educación política se constituyeron en ámbitos de disputa entre los modelos de estado, sociedad y economía que se hallaban en pugna desde inicio del siglo XX. 


\section{La radicalización de la participación política juvenil durante el tercer peronismo (1973-1976)}

Tras dieciocho años de alternancia entre gobiernos dictatoriales y democráticos, el peronismo vuelve al poder en 1973. En este contexto las instituciones educativas fueron escenario de procesos de radicalización política, de creciente presencia sindical y de distintas iniciativas de apertura de la participación de los adolescentes y jóvenes que conformaban el estudiantado, por ejemplo, a través de centros de estudiantes o del ingreso a la militancia político partidaria.

En ese marco, la nueva materia dedicada a la educación política, Estudio de la Realidad Social Argentina (ERSA) tenía como propósito crear en los estudiantes el interés por la realidad social, política y económica. Según el Ministerio de Educación de 1974 “... tendía a promover entre los estudiantes la aptitud y la metodología para el análisis crítico de la sociedad, para la investigación, el diálogo y la creatividad" (Lamas, 2013, p. 7). La organización de los contenidos políticos de ERSA era la siguiente: en primer año se enseñaba esencialmente el análisis crítico de la situación nacional, en segundo año se estudiaba la sociedad de clases en América Latina y en tercero el contexto geopolítico internacional. El marco teórico y pedagógico eran la teoría de la dependencia, por un lado, y la pedagogía de la liberación de Paulo Freire, por el otro.

Los contenidos estaban organizados alrededor de un eje denominado "soberanía o dependencia” y se desarrollaba en cuatro áreas interrelacionadas: economía, política, sociedad y cultura. Los saberes se graduaron de lo más cercano a lo más lejano en términos territoriales, de primero a tercer año del nivel medio, así: primero, Argentina como país; segundo, Argentina en Latinoamérica; tercero, Argentina en el mundo.

El modelo de sociedad que se plantea es el de una sociedad participativa, con un amplio protagonismo de las organizaciones, desde las cívico-barriales y los centros estudiantiles hasta las Naciones Unidas. Es decir, la participación democrática no se restringe al ejercicio del sufragio, sino que conforma una compleja red de relaciones sociales solidarias. Se inicia con la actividad de los adolescentes en los centros estudiantiles en las escuelas, continúa con el compromiso barrial de los jóvenes y la actuación de hombres y mujeres en juntas vecinales y sociedades de fomento, para tener su expresión más acabada en la elección del gobierno nacional. Se dedica un apartado especial, por primera vez, a los partidos políticos como canales de la participación ciudadana y formadores de la representación política. (Ministerio de Educación y Cultura de la Nación, Educ.ar, s.f.)

Hacia 1975, en un contexto de creciente convulsión política nacional, el control y el disciplinamiento se fortalecían como la contracara de la creciente participación política juvenil en el marco de las movilizaciones estudiantiles latinoamericanas y mundiales. La 
materia ERSA fue eliminada del plan de estudios y fue reconvertida en educación cívica, mientras estudiantes y docentes comenzaban a sufrir el impacto de la represión, la tortura, los secuestros y las desapariciones. El nacionalismo autoritario retornaba a la escena nacional reorientando la educación política de adolescentes y jóvenes bajo sus principios.

\section{La represión sobre la participación juvenil como política de estado durante la dictadura militar (1976-1983)}

El 24 de marzo de 1976 se impusó el último golpe de Estado cívico-militar en Argentina. Según los principios autoritarios de la dictadura, la educación debía ser controlada, ya que, a través de ella se adoctrinarían los subversivos que atentarían contra la patria. La escuela secundaria y las universidades quedaron en el foco de las estrategias de control, “... primer objetivo de esta política era la persecución ideológica pero también una militarización de la educación en la que prevaleciera la obediencia, el orden, el control, las jerarquías, los actos escolares pautados, los uniformes y el pelo corto" (De Amézola, 2008, p. 45). Además, sectores de la sociedad civil acompañaron y legitimaron esta persecución sobre docentes y estudiantes, destacándose las orientaciones de pedagogos que fomentaban la formación de individuos acríticos y pasivos (Kaufmann y Doval, 1997). Por otro lado, controlar los contenidos de los cursos de educación política y de historia fue un objetivo de máxima, ya que se pensaba que allí se formaba la ideología marxista y se trazaban los saberes necesarios para la construcción de la subversión. Como consecuencia, la dictadura trajo un reforzamiento de contenidos tradicionales tendientes a fomentar la exaltación de la nación y una vuelta a las estrategias de enseñanza tradicionales que permitirían el control de los sujetos, presumiendo que ciertas destrezas cognitivas centradas en los sujetos favorecerían el pensamiento crítico y autónomo (Jorquera, 2019).

Esta dictadura, postulará, además, la necesidad de introducir la formación técnica y laboral en la Escuela Media, en pos de desalentar las expectativas de ingreso a la educación superior, la profesionalización creciente de la juventud y la formación del carácter político que se forjaba en las universidades. Esta revalorización del bachillerato, condujo a cambios curriculares que expresaban una perspectiva conservadora mediante la introducción de formación moral y cívica. En tanto la participación política de los alumnos dentro de los Centros de Estudiantes - asambleas de estudiantes formadas para la deliberación de sus propios asuntos- fue alentada desde 1973 tras la vuelta del peronismo al gobierno, en 1975, tras la muerte de Perón, el Ministerio disuelve las organizaciones estudiantiles anticipando una situación que perdurará durante toda la dictadura 
e incluso durante el primer gobierno democrático en el periodo 1983-1989. Para garantizar que los estudiantes no promuevan el debate político, la Circular 6 de enero de 1975 del Ministerio de Cultura y Educación de la Nación prohibirá a los alumnos entrar en aulas distintas a las que cada estudiante tiene asignadas y permanecer en ellas durante el recreo. Así, mientras la última dictadura cívico militar consolidaba su carácter represivo sobre el conjunto de la sociedad argentina, en materia educativa desplegaba con énfasis mecanismos violentos en pos del control sobre aquellas juventudes que, desde hace décadas, se venían alimentando de la creciente participación política, la formación ciudadana y la resistencia tomada desde el modelo de acción de los sectores trabajadores. El secuestro, la tortura, la desaparición y la muerte doblegará a las juventudes organizadas bajo un plan sistemático de represión y aniquilamiento que los tendrá a ellos como uno de sus principales blancos.

\section{La reconstrucción de la democracia y la educación política (1983-2020)}

Con la vuelta de la democracia hacia 1983, el giro educativo consistió en el trabajo centrado en la construcción de principios democráticos intentando quitar de los ámbitos educativos aquellos resabios de la educación autoritaria, mediante, por ejemplo, la incorporación de la materia educación cívica en el nivel secundario. La sociedad argentina clamaba por una nueva era donde la memoria, la verdad y la justicia se debían erigir como sólidos pilares para revisar el pasado reciente. La educación política se convertía en el bastión para la transición democrática. No obstante, las tradiciones de enseñanza acuñadas en las experiencias represivas recientes impidieron la transformación total de la educación política, consolidándose una educación cívica que mientras buscaba revitalizar concepciones políticas como democracia, estado de derecho, división de poderes y república, lo hacía desde estrategias de enseñanza instruccionales y repetitivas que, más que producir un cambio hacia la conciencia democrática, recordaban al modelo didáctico de la dictadura.

A cien años de realizado el Primer Congreso Pedagógico y después de haber sufrido la peor dictadura de la historia argentina, la ciudadanía se planteaba una revisión de aquellos valores que condujeron a la sociedad a recibir de forma resiliente a un gobierno que masacró, persiguió y torturó a todos los opositores políticos. En palabras de Puiggrós durante el año 1987:

El debate sobre el carácter democrático o autoritario de la educación cruza toda la historia de la educación argentina y latinoamericana...si consideramos que el sistema escolar es solamente una parte del sistema educativo de la sociedad y que éste abarca el conjunto de 
procesos educativos que se desarrollan en todas las instituciones y en todos los vínculos sociales, la reflexión sobre el papel de la educación en la construcción de vínculos democráticos o autoritarios cobra aún más importancia. Este es un tema de primer orden en el proceso de construcción democrática de varios países latinoamericanos y particularmente en Argentina. (Díaz y Kaufman, 2006, p. 155)

A pesar que el debate iniciado por el Segundo Congreso Pedagógico Nacional -que tuvo lugar entre 1984 y 1988- se centró en la reconstrucción de los valores democráticos, los resultados de las discusiones nunca se hicieron efectivos dado que Argentina entró en la primera gran crisis de deuda externa en 1989 obturando los debates abiertos por la transición democrática.

\section{La educación como un bien de mercado durante el periodo neoliberal (1989-2001)}

En 1989 asume la presidencia Carlos Saúl Menem, quien contrariamente a sus promesas de campaña de un nuevo pacto productivo y distributivo, impondrá los lineamientos políticos, sociales y económicos trazados por el Consenso de Washington. En el marco de un férreo plan neoliberal, el menemismo impulsará una reforma educativa, acorde a los requerimientos de los organismos internacionales de crédito y el paradigma de la tecnocracia educativa, bajo el lema de la calidad y la equidad en el marco del cambio tecnológico y de la sociedad del conocimiento. Como parte del plan de reforma del Estado y privatización, la reforma educativa propondrá la descentralización de la educación provocando la redefinición de la relación entre el Estado y la ciudadanía. La educación se constituyó en uno de los campos donde la racionalidad tecnocrática neoliberal orientó el proceso de reforma, que incluyó: la total descentralización y federalización del sistema educativo, a cargo ahora de las distintas provincias - salvo las universidades que continúan siendo nacionales-; la centralización y establecimiento de los Contenidos Básicos Comunes y la orientación pedagógica general en manos del Ministerio de Educación y Cultura de la Nación; el reemplazo de la histórica escuela primaria por la Educación General Básica (EGB), que extiende de siete a nueve años la escolaridad obligatoria y; la transformación de la escuela secundaria hacia la educación polimodal de solo tres años de duración y con un fuerte compromiso en su oferta de terminalidades para el mercado laboral. De este modo, el menemismo lejos de definir a la educación pública como un derecho la convirtió en un bien de cambio.

En este contexto, se instituyó en 1993 la Ley Federal de Educación 24 195, que en su Artículo Nº 1 establecerá: 
El derecho constitucional de enseñar y aprender queda regulado, para su ejercicio en todo el territorio argentino, por la presente ley que, sobre la base de principios, establece los objetivos de la educación en tanto bien social y responsabilidad común, instituye las normas referentes a la organización y unidad del Sistema Nacional de Educación, y señala el inicio y la dirección de su paulatina reconversión para la continua adecuación a las necesidades nacionales dentro de los procesos de integración.

No obstante, si bien el Artículo No. 6 de la Ley Federal 24195 de 1993 expresa que,

El sistema educativo posibilitará la formación integral y permanente del hombre y la mujer, con vocación nacional, proyección regional y continental y visión universal, que se realicen como personas en las dimensiones cultural, social, estética, ética y religiosa, acorde con sus capacidades, guiados por los valores de vida, libertad, bien, verdad, paz, solidaridad, tolerancia, igualdad y justicia. Capaces de elaborar, por decisión existencial, su propio proyecto de vida. Ciudadanos responsables, protagonistas críticos, creadores y transformadores de la sociedad, a través del amor, el conocimiento y el trabajo. Defensores de las instituciones democráticas y del medio ambiente.

En términos concretos, aquello que la Ley dice sostener fue obstaculizado por las políticas de mercado que incidieron de manera drástica en el goce de derechos básicos para la vida. Respecto de la educación política, la propuesta neoliberal se desarrollará a través de la materia formación ética y ciudadana, organizada en los siguientes bloques: Bloque 1: persona. Bloque 2: valores. Bloque 3: normas sociales. Bloque 4: procedimientos generales. Bloque 5: actitudes generales.

En términos de resultados, la materia continuó enseñándose bajo el paradigma instruccional y el enfoque teórico de la disciplina política, impidiendo la problematización de los contenidos bajo dinámicas activas. Ello se vio profundizado por el hecho de que los principios sostenidos por la norma - tales como justicia, derechos, igualdad y solidaridad, entre otros- fueron obstruidos por las políticas de ajuste del Estado y la imposición de las reglas del mercado, dejando a millones de adolescentes y jóvenes en situación de vulnerabilidad socio educativa, cultural y material.

Como resultado no solo no se concretó la participación juvenil a través de la educación política, sino que la realidad de la sociedad excluyente (Svampa, 2005), obturó toda posibilidad a la garantía de derechos fundamentales, mientras se desplegaban políticas compensatorias que no lograban equilibrar la vulnerabilidad de amplios sectores de la sociedad

La resistencia a esta Ley (1993) fue tan importante que tanto docentes como alumnos comienzan a organizarse para frenar el embate del gobierno contra la escuela pública que progresivamente era desfinanciada a la par que se recortaba la cantidad de horas aplicadas a la formación básica. 
La crisis educativa resultante, provocó la movilización de docentes y estudiantes, quienes gestaron la Carpa Blanca Docente frente al Congreso Nacional desde abril de 1997 hasta el 30 de diciembre de 1999. La carpa, organizada por la Central de Trabajadores de la Educación de la República Argentina (CTERA) se convirtió en un símbolo nacional que sostuvo la lucha docente por robustecer salarios, mejorar las condiciones de trabajo y avanzar en el financiamiento de la educación pública.

Figura 1. La Carpa Blanca Docente instalada en abril de 1997.

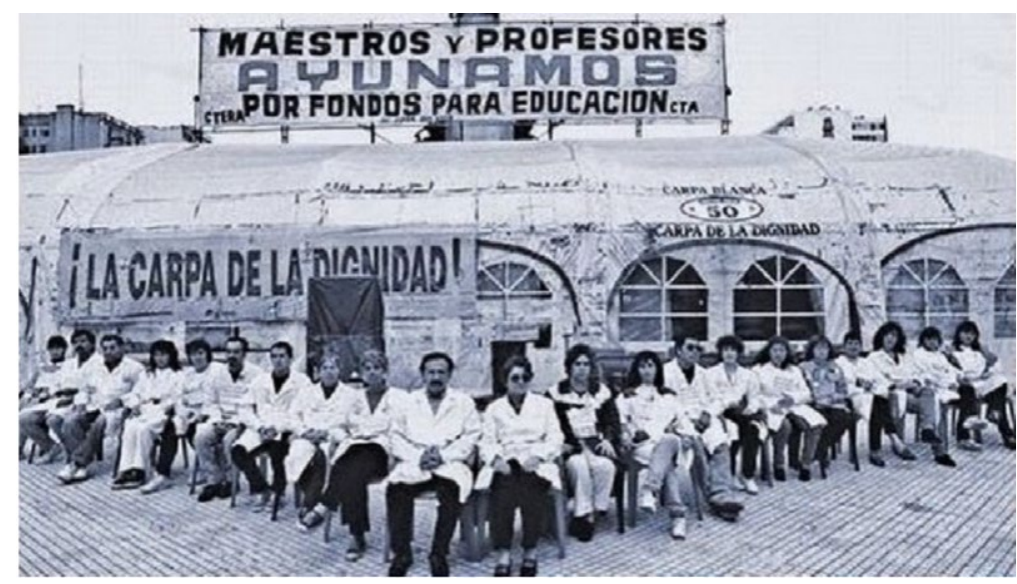

Fuente: Ciudad de Buenos Aires, 1997. Agencia de Noticias Formosa (s.f.)

De esta manera, si bien las políticas neoliberales buscaban la despolitización de la sociedad, las medidas de ajuste en educación tuvieron como consecuencia la organización de la sociedad civil a través de sus entidades tradicionales de representación -como sindicatos y gremios-y de organización de movimientos sociales y territoriales.

Asimismo, a pesar que el fortalecimiento de los derechos juveniles no se consideraba relevante, durante las presidencias de Menem se desarrolló un proyecto denominado el "voto pendex", término banal aplicado al voto juvenil. La intención era aprovechar el voto adolescente como apoyo de un proyecto de consolidación del poder de Menem cuando aspiraba a ser reelecto y la Constitución aún no lo habilitaba para obtener un segundo mandato.

La decisión de hacer votar a los adolescentes fue lanzada por el entonces Ministro del Interior de Menem, Gustavo Béliz, al amparo de lo que se conoció como "reforma política”, en medio del escándalo de corrupción desatado en Italia por el caso Mani pulite - manos limpias-, que había involucrado a funcionarios del Gobierno argentino. 
Béliz, cuya mayor preocupación era la corrupción, defendió aquella reforma política integral que incluía al "voto pendex" en un conjunto de medidas tales como las internas abiertas en los partidos, el blanqueo de aportes a partidos y candidatos, un sistema de tachas para mejorar las listas sábana, y que los partidos no desaparecieran por falta de votos.

No obstante, las encuestas anunciaban que el $60 \%$ de la población, entre ella los adolescentes sospechaban sobre el uso del voto juvenil. A la sospecha no le faltaban razones: se trataba de 1300000 votos en danza a los que el proyecto quería dar carácter optativo, pero con los que Menem planeaba arrasar en las elecciones de 1995. La oposición a la propuesta era amplia y fue dejada de lado meses después.

En paralelo al voto a los 16 años, se inicia el debate sobre la baja de la edad de imputabilidad. En general, una propuesta de carácter liberal -la del voto juvenil- y la otra conservadora - la baja de la imputabilidad - fueron lo que prefiguraba la necesidad de un cambio profundo en la perspectiva de análisis de los jóvenes como sujetos sin derechos o sujetos con derechos.

Mientras las políticas neoliberales continuaban su profundización hacia 1999 durante el gobierno de De la Rúa, comenzaba a producirse la peor crisis social, política y económica del país, que tendrá como punto de eclosión el estallido social y la resistencia de diciembre de 2001, conocido como el "Argentinazo". Luego de más de una década de políticas de retroceso social y material de la ciudadanía, la sociedad civil tomará las calles reclamando "que se vayan todos".

\section{Educación política y ejercicio pleno de la ciudadanía durante la década kirchnerista (2003-2015)}

Transcurrida la crisis de 2001 y como producto del nuevo contexto socioeconómico caracterizado por políticas de desarrollo económico interno y de recuperación del empleo, en un marco de garantía de los derechos sociales, comienza a regir en Argentina la Ley Nacional de Educación 26206 de 2006, dando inicio a un nuevo proceso de reforma educativa de alcance nacional que establece "el derecho de enseñar y aprender" como un derecho social. Así el texto de la Ley (2006) expresa en su Artículo No. 1 lo siguiente:

La presente ley regula el ejercicio del derecho de enseñar y aprender consagrado por el artículo 14 de la Constitución Nacional y los tratados internacionales incorporados a ella, conforme con las atribuciones conferidas al Honorable Congreso de la Nación en el artículo 75, incisos 17, 18 y 19, y de acuerdo con los principios que allí se establecen y los que en esta ley se determinan. 
Mientras que en su artículo $\mathrm{N}^{\circ}$. 2 expresa el rol esencial del estado como garante de tal derecho: "La educación y el conocimiento son un bien público y un derecho personal y social, garantizados por el Estado".

Se abre un nuevo periodo donde la política educativa instituye al Estado argentino como garante y, en un marco donde la educación es entendida como un derecho social, el enfoque de la inclusión educativa tendrá un protagonismo central en las políticas públicas educativas orientadas a garantizar el "derecho de enseñar y aprender" a nivel nacional y en sus adecuaciones provinciales.

En torno al año 2003 en un marco de redistribución de bienes materiales y culturales, cobra relevancia el propósito de la inclusión socioeducativa que surge tras el diagnóstico y la comprensión de las raíces de la "nueva cuestión social" a partir del repliegue y los límites del estado providencia (Rosanvallon, 1995), en un marco de debilitamiento de la sociedad salarial y del trabajo como condición de existencia (Castel, 1997) que caracterizan a las últimas décadas del siglo XX y los inicios del siglo XXI.

Se plantea el desafío de la justicia social y las prácticas democráticas en educación donde el propósito de "inclusión educativa", entendida como "un proceso de abordaje y respuesta a la diversidad en las necesidades de todos los alumnos a través de la creciente participación en el aprendizaje, las culturas y las comunidades, y de la reducción de la exclusión dentro y desde la educación" (Opertti 2008, p. 14), requiere de políticas educativas diversas, específicas y direccionadas para su consecución.

Como consecuencia de los propósitos de inclusión socio educativa de adolescentes y jóvenes, la nueva política pública en educación coloca el foco en la educación política y la necesidad de transformación de los paradigmas escolares de la enseñanza de la política en el país.

Desde la misma Ley de Educación Nacional 26206 de 2006 en su artículo Nº. 92 se profundizaron los propósitos de la educación política, estableciendo el fortalecimiento de la identidad nacional y la democracia, la construcción de la memoria histórico-social, la defensa de los derechos humanos, la integración latinoamericana focalizando en la región Mercosur, los Derechos de Niños, Niñas y adolescentes y la diversidad cultural de los Pueblos Originarios y sus derechos, junto con relaciones basadas en la igualdad, la solidaridad, el respeto entre los sexos y contra la discriminación de la mujer. Del mismo modo, en el artículo No. 30 de la misma Ley se instituye que "la Educación Secundaria en todas sus modalidades y orientaciones tiene la finalidad de habilitar a los/ las adolescentes y jóvenes para el ejercicio pleno de la ciudadanía, para el trabajo y para la continuación de estudios". 
En ese marco, las veintitrés provincias que componen la República Argentina junto a la Ciudad Autónoma de Buenos Aires, adecuan sus planes de estudios en función de las nuevas políticas en educación y en pos de la incorporación de la educación política bajo el paradigma de la inclusión, la participación ciudadana democrática y el enfoque de los Derechos Humanos. Como caso particular, la Provincia de Buenos Aires emprende la adecuación de sus planes de estudios creando áreas curriculares para la enseñanza de la educación política destinada a niños, niñas y jóvenes bonaerenses.

El nuevo esquema curricular para la educación ciudadana en la Provincia -que funcionará como modelo para la adecuación en todo el país- estará compuesta por los siguientes espacios curriculares: construcción de ciudadanía para $1^{\circ}, 2^{\circ}$ y $3^{\circ}$ años del Ciclo Básico de la escuela secundaria y las materias salud y adolescencia para $4^{\circ}$ año, política $y$ ciudadanía para $5^{\circ}$ año y trabajo y ciudadanía para $6^{\circ}$ año del Ciclo Superior de la educación secundaria. Bajo dicho esquema curricular -que se incorpora al esquema de materias disciplinares- el Estado provincial mediante el sistema educativo asume,

El deber ético y público de educar a las nuevas generaciones como sujetos políticos, con la convicción de que un fin de la Educación Secundaria es la construcción de sujetos autónomos que hagan valer sus derechos y cumplan sus responsabilidades en ámbitos como la familia, el aula, la escuela, la comunidad y el trabajo. (DGCyE, 2007, p. 21)

Bajo el paradigma de la ciudadanía activa, promotora de prácticas democráticas basadas en el enfoque de los Derechos humanos y una didáctica ciudadana sustentada en la enseñanza desde las mismas prácticas sociales, se buscó, también, la democratización de las instituciones educativas y del aula como espacios de ejercicio del derecho y la responsabilidad de enseñar, y el derecho y la responsabilidad de aprender.

El nuevo paradigma de la enseñanza política en Argentina basado en el ejercicio activo y crítico de la ciudadanía implicó la incorporación de una serie de principios tales como concebir a niños, niñas, adolescentes y jóvenes como sujetos de derechos, es decir como ciudadanas y ciudadanos, entender a la ciudadanía misma como un conjunto de prácticas que definen a un sujeto como miembro de una sociedad, en su relación con otros sujetos y con el Estado, reconocer que las prácticas de niños, niñas, adolescentes y jóvenes son prácticas ciudadanas en tanto son modos de inscribirse, insertarse o incluirse en la sociedad, analizar críticamente los contextos socioculturales y las condiciones de diversidad y desigualdad que lo constituyen desde una perspectiva de derechos y proyectar acciones colectivas de ejercicio y exigibilidad de derechos y responsabilidades.

En correlación con ello, el Diseño Curricular para Construcción de Ciudadanía de la Provincia de Buenos Aires indica que: 
No se apela a la concepción de formar ciudadanos "en" o "para el" futuro, posdatando la participación activa de los sectores jóvenes de la sociedad en espacios donde son actores fundamentales. Por el contrario, se interpela a todos los actores institucionales y sociales en el contexto sociocultural actual para comprometerlos en acciones de interrelación, de asunción de responsabilidades, de generación de vínculos y lazos sociales, asumiendo responsabilidades con los otros, que tomen la forma de proyectos colectivos posibles de llevar a cabo en el transcurso de los ciclos lectivos y en el marco del currículum obligatorio de la educación secundaria. (DGCE, 2007, p. 26)

El nuevo paradigma de educación política en Argentina fue el correlato de un paradigma normativo de mayor envergadura basado en la Doctrina de la Protección Integral fundado en la concepción de que todos los niños, niñas, adolescentes y jóvenes son sujetos de derechos y por este carácter de universalidad todos son destinatarios del mismo, mientras las políticas diseñadas desde esta concepción integral deben ser políticas inclusivas. Así, si bien los niños, niñas, adolescentes y jóvenes como seres humanos quedan comprendidos en las declaraciones internacionales de derechos humanos, la historia reciente del país consagrará sus derechos particulares a través la Ley Nacional 26061 de 2005 de "Protección Integral de los Derechos de las niñas, niños y adolescentes".

Dado que la educación ciudadana propuesta por la legislación y los currículums apelan a la construcción y despliegue de prácticas sociales y situadas donde los niños, niñas, adolescentes y jóvenes son los actores primordiales, las propuestas didácticas para la formación ciudadana se proyectan en torno a estrategias de problematización, o sea, como espacios reales de ejercicio de la ciudadanía. No se trata de instruir ciudadanos, sino de ejercer la ciudadanía a través de la escuela, de reconocerse como ciudadana y ciudadano, de luchar para ser reconocidos en una agenda de expansión de derechos (DGCE, 2007).

Para tal fin, la organización curricular de la educación ciudadana propone una serie de "ámbitos para la construcción de la ciudadanía" (DGCE, 2007) para los tres primeros años de la educación secundaria como fundamento para el diseño de proyectos educativos. Los ámbitos para la construcción de la ciudadanía son: ambiente; arte; comunicación y tecnologías de la información; Estado y política; identidades y relaciones interculturales; recreación y deporte; salud, alimentación y drogas; sexualidad y género; trabajo.

Las dos materias para la formación ciudadana del Ciclo Superior de la educación secundaria - política y ciudadanía en $5^{\circ}$ año y Trabajo y ciudadanía en $6^{\circ}$ año- continúan la estrategia del pleno ejercicio de la ciudadanía, pero con la incorporación de un marco teórico conceptual que se ha de desarrollar de manera gradual y mediante una dinámica de complejización de la teoría social. No obstante, el principal aprendizaje de la ciudadanía continúa realizándose a través del análisis, la experiencia y la sistematización de la práctica de los derechos y las responsabilidades, esto es, el ejercicio de la ciudadanía como 
sujeto político en tanto ciudadano pleno. De tal modo, los saberes que se abordaran en el espacio curricular de política y ciudadanía de $5^{\circ}$ año se distribuyen estratégicamente en unidades, a saber: política; Estado y gobierno; ciudadanía, participación y organización política; derechos humanos y democracia.

Mientras que para el espacio curricular de trabajo y ciudadanía para $6^{\circ}$ año se estructuran los saberes en torno a la condición de los trabajadores y del mundo del trabajo mediante una serie de unidades enunciadas de la siguiente manera: la organización del trabajo en las sociedades capitalistas; derechos y obligaciones laborales; los derechos de los trabajadores; la búsqueda de trabajo; la condición juvenil; la participación política de los jóvenes; sistema educativo y participación estudiantil.

El caso de la materia salud y adolescencia diseñada para el $4^{\circ}$ año de la educación secundaria merece especial atención dado su propósito de abordar las prácticas de salud como una dimensión particular de las prácticas sociales y políticas inherentes a la formación ciudadana. Así, si bien la materia introduce la mirada biológica y psicoanalítica en pos de las prácticas de salud, también incorpora el nuevo paradigma de política educativa que concibe a los niños, niñas, adolescentes y jóvenes como sujetos de derecho dirigiéndose al cuidado de la salud en torno a prácticas responsables sobre el cuerpo y la psique de cada etapa evolutiva y la salud concebida como un derecho básico de las personas. No obstante, se promueve el cuidado y las prácticas de una vida saludable, salud y adolescencia refiere al paradigma educativo de la Educación Sexual Integral donde la Ley 26150 de 2006, "Programa Nacional de Educación Sexual Integral", orienta la educación de niños, niñas, adolescentes y jóvenes en la ya mencionada práctica de salud ciudadana como un criterio transversal. En ese marco, la materia se compone de módulos diseñados para la problematización, estructurados de la siguiente forma: la definición actual de salud/enfermedad; los jóvenes y los procesos de salud/enfermedad; la educación sexual integral; el derecho a la salud; espacios de participación juvenil en la promoción y prevención de la salud.

\section{Conclusiones}

Como resultado de una experiencia histórica fragmentada por la puja entre modelos sociopolíticos autoritarios y democráticos u oligárquicos y populares, y procesos materiales basados en el mercado o en la distribución social, hemos verificado que la educación política en la República Argentina ha sufrido mutaciones constantes y contrapuestas, mientras la escuela, a pesar de la transformación de su currículum y de sus planes de estudio, continúa siendo la institución legítima y capaz para la consecución de los propósitos de la educación política delineada por el Estado. 
La República Argentina post neoliberal, inicia un periodo de restitución legal normativo y social de los derechos, diseñado y direccionado primordialmente por el Estado nacional y, luego, por los Estados provinciales, que acompañado de nuevos paradigmas jurídicos y educativos en torno a la niñez, la adolescencia y la juventud, transforman las prácticas de la educación política en las escuelas mediante estrategias críticas ancladas en la realidad social de los sujetos y basadas en el ejercicio pleno e integral de la ciudadanía y la construcción gradual del marco teórico conceptual propio de la teoría social.

Finalmente, las políticas educativas específicas como las que hemos recorrido, sumado a una batería de políticas públicas destinadas a movilizar y mejorar la vida durante la infancia y la juventud - el logro del voto a los 16 años y la Ley de Protección integral de los Derechos de niños, niñas y adolescentes, entre otros-crearán en Argentina un nuevo paradigma político sustentado en un estado de derecho que culminará otorgando legitimidad y posteridad al protagonismo sociopolítico de adolescentes y jóvenes.

\section{Referencias}

[1] Agencia de Noticias Formosa. (s.f.). Ayunante de la histórica Carpa Blanca recordó la gesta. https://agenfor.com.ar/ayunante-de-la-historica-carpa-blanca-recordo-la-gesta/

[2] Castel, R. (1997). La sociedad salarial. Las metamorfosis de la cuestión social. Paidós.

[3] Consejo Nacional de Educación, El monitor de la educación Común, Año 34 No. 528, 31 de Diciembre de 1916.

[4] Consejo Nacional de Educación, El monitor de la educación Común, Año 35 No. 536, 31 de Agosto de 1917.

[5] Cora, G. D. y Rodríguez, M. (2015). Construyendo la "Nueva Argentina". Cultura ciudadana y la consolidación de la política educativa del peronismo (1952-1955). Espacio, Tiempo y Educación, 2(1), 187-205. http://dx.doi.org/10.14516/ete.2015.002.001.010

[6] De Amézola, G. (2008). Esquizohistoria. La historia que se enseña en la escuela, la que preocupa a los historiadores y una renovación posible de la historia escolar. Del Zorzal.

[7] Díaz, N. y Kaufmann, C. (2006). El II Congreso Pedagógico Nacional (1984-88) a través de los diarios regionales El Litoral y El Diario. Ciencia, Docencia y Tecnología, 17(32), 153-177. https:/www.redalyc.org/pdf/145/14503205.pdf

[8] Dirección General de Cultura y Educación (DGCE). (2007). Diseño curricular para la educación secundaria: construcción de ciudadanía: $1^{\circ}$ a $3^{\circ}$ año, coordinado por Ariel Zysman y Marina Paulozzo. http://abc.gob.ar/secundaria/dc_123

[9] Escudé, C. (1990). El fracaso del proyecto argentino. Educación e ideología. Instituto Torcuato Di Tella. 
[10] Jorquera, M. I. (2019). Las estrategias de enseñanza en las clases de historia: cambios y permanencias en las escuelas secundarias de La Matanza [tesis de maestría, FlacsoArgentina]. Repositorio institucional. http://hdl.handle.net/10469/16246

[11] Kaufmann, C. y Doval, D. (1997). Una pedagogía de la renuncia. El perennialismo en Argentina (1976-1983). Cuadernos-UNER.

[12] Lamas, M. I. (2013). La asignatura ERSA (Estudios de la realidad social argentina): un aporte al estudio de la educación ciudadana en la década del 70 a partir de un libro de texto [Presentación de ponencia]. I Jornadas Norpatagónicas de Experiencias Educativas en Ciencias Sociales para la Escuela Secundaria, II Jornadas Provinciales de Geografía, Ciencias Sociales y Educación, Neuquén, Luis Beltrán, Río Negro, Argentina.

[13] Ministerio de Educación v Cultura de la Nación. (1975). Resolución 41 de 1975. Circular $n^{\circ} 6$.

[14] Ministerio de Educación y Cultura de la Nación. Educ.ar (s.f.). La vuelta del peronismo al gobierno. ERSA. Formación ética y ciudadana. Núcleo teórico. Tradiciones de enseñanza. Disponible en: https://cdn.educ.ar/dinamico/UnidadHtml_get_14b34df81fdf-49ff-97d6-180592d35bd8/110352/index.html

[15] Opertti, R. (2008). Inclusión Educativa: El Camino del Futuro. Un desafío para compartir [Presentación de ponencia]. Taller Internacional sobre Inclusión Educativa América Latina - Regiones Cono Sur y Andina, Montevideo, Uruguay.

[16] Presidencia de la Nación Argentina. (1884, 26 de junio). Ley 1420 de 1884. Educación Común. http://www.infoleg.gob.ar/

[17] Presidencia de la Nación Argentina. (1905, 17 de octubre). Ley 4874 de 1905. Ley Láinez, de extensión de la educación pública al creciente territorio nacional. http:// www.infoleg.gob.ar/

[18] Presidencia de la Nación Argentina. (1912, 26 de marzo). Ley 8871 de 1912. Elecciones nacionales. http://www.infoleg.gob.ar/

[19] Presidencia de la Nación Argentina. (1943, 31 de diciembre). Decreto 18411 de 1943. Disponiendo que en todas las escuelas públicas de enseñanza primaria, post primaria, secundaria y especial, la enseñanza de la religión católica será impartida como materia ordinaria de los respectivos planes de estudios y creando la Dirección General de Instrucción Religiosa. http://www.bnm.me.gov.ar/giga1/normas/14066.pdf

[20] Presidencia de la Nación Argentina (1993, 14 de abril). Ley 24195 de 1993. Ley Federal de Educación. http://www.infoleg.gob.ar/

[21] Presidencia de la Nación Argentina. (2005, 21 de octubre). Ley 26061 de 2005. Protección integral de los derechos de las niñas, niños y adolescentes. https://siteal.iiep.unesco.org/ bdnp/6/ley-26061-proteccion-integral-derechos-ninas-ninos-adolescentes 
[22] Presidencia de la Nación Argentina. (2006, 4 de octubre). Ley 26150 de 2006. Programa Nacional de Educación Sexual Integral. http://www.infoleg.gob.ar/

[23] Presidencia de la Nación Argentina. (2006, 14 de diciembre). Ley 26206 de 2006. Educación Nacional. http://www.infoleg.gob.ar/

[24] Puiggrós, A. (2003). Qué pasó en la educación argentina. Breve historia desde la conquista hasta el presente. Galerna.

[25] Rosanvallon, P. (1995). La nueva cuestión social. Repensar el Estado providencia. Manantial.

[26] Svampa, M. (2005). La sociedad excluyente. La Argentina bajo el signo del neoliberalismo. Taurus.

\section{Anexos}

Anexo 1. Ministerios de Educación de la Nación, ministros a cargo y presidencia correspondiente a través del tiempo

\begin{tabular}{|c|c|c|c|}
\hline & $\begin{array}{l}\text { Ministro de } \\
\text { educación }\end{array}$ & Periodo & Presidencia \\
\hline 1 & Ivanisevich & 11 de marzo de 1949 - 11 de mayo de 1950 & \multirow{4}{*}{ Perón } \\
\hline 2 & $\begin{array}{l}\text { Armando Méndez } \\
\text { San Martín }\end{array}$ & 21 de junio de 1950 - 29 de junio de 1955 & \\
\hline 3 & $\begin{array}{l}\text { Francisco Marcos } \\
\text { Anglada }\end{array}$ & 1 de julio de 1955 - 21 de septiembre de 1955 & \\
\hline 4 & $\begin{array}{l}\text { Atilio Dell'Oro } \\
\text { Maini }\end{array}$ & $\begin{array}{l}23 \text { de septiembre de } 1955 \text { - } \\
13 \text { de noviembre de } 1955\end{array}$ & \\
\hline 5 & $\begin{array}{l}\text { Atilio Dell'Oro } \\
\text { Maini }\end{array}$ & 13 de noviembre de 1955 -17 de mayo de 1956 & \multirow{5}{*}{$\begin{array}{l}\text { Golpe de } \\
\text { Lonardi y } \\
\text { Aramburu }\end{array}$} \\
\hline 6 & Carlos Adrogué & 8 de junio de 1956 - 25 de enero de 1957 & \\
\hline 7 & $\begin{array}{l}\text { Acdel Ernesto } \\
\text { Salas }\end{array}$ & 25 de enero de 1957 - 1 de mayo de 1958 & \\
\hline 8 & $\begin{array}{l}\text { Luis Rafael } \\
\text { Mac Kay }\end{array}$ & 1 de mayo de 1958 - 26 de marzo de 1962 & \\
\hline 9 & Miguel Sussini & 26 de marzo de 1962 - 29 de marzo de 1962 & \\
\hline 10 & Miguel Sussini & 29 de marzo de 1962 - 19 de octubre de 1962 & \multirow{3}{*}{ Guido } \\
\hline 11 & $\begin{array}{l}\text { Alberto } \\
\text { Rodríguez Galán }\end{array}$ & 11 de octubre de 1962 - 15 de mayo de 1963 & \\
\hline 12 & $\begin{array}{l}\text { José Mariano } \\
\text { Astigueta }\end{array}$ & 15 de mayo de 1963 - 12 de octubre de 1963 & \\
\hline 13 & $\begin{array}{l}\text { Carlos Alconada } \\
\text { Aramburú }\end{array}$ & 12 de octubre de 1963 - 28 de junio de 1966 & Illia \\
\hline
\end{tabular}




\begin{tabular}{|c|c|c|c|}
\hline & $\begin{array}{l}\text { Ministro de } \\
\text { educación }\end{array}$ & Periodo & Presidencia \\
\hline 14 & $\begin{array}{l}\text { Carlos María } \\
\text { Gelly y Obes }\end{array}$ & 28 de junio de 1966 - 4 de junio de 1967 & \multirow{3}{*}{$\begin{array}{l}\text { Golpe de } \\
\text { Ongania }\end{array}$} \\
\hline 15 & $\begin{array}{l}\text { José Mariano } \\
\text { Astigueta }\end{array}$ & 4 de junio de 1967 - 23 de octubre de 1969 & \\
\hline 16 & $\begin{array}{l}\text { Dardo Pérez } \\
\text { Guilhou }\end{array}$ & 23 de octubre de 1969 - 8 de junio de 1970 & \\
\hline 17 & José Luis Cantini & 18 de junio de 1970 - 23 de marzo de 1971 & Levingston \\
\hline 18 & Gustavo Malek & 26 de marzo de 1971 - 25 de mayo de 1973 & Lanusse \\
\hline 19 & $\begin{array}{l}\text { Jorge Alberto } \\
\text { Taiana }\end{array}$ & 13 de julio de 1973 - 14 de agosto de 1974 & \multirow{3}{*}{$\begin{array}{l}\text { Campora } \\
\text { Perón } \\
\text { Isabel } \\
\text { Perón }\end{array}$} \\
\hline 20 & Oscar Ivanissevich & 14 de agosto de 1974-11 de agosto de 1975 & \\
\hline 21 & Pedro J. Arrighi & 11 de agosto de 1975 -24 de marzo de 1976 & \\
\hline 22 & César Guzzetti & 24 de marzo - 29 de marzo de 1976 & \multirow{6}{*}{$\begin{array}{l}\text { Dictadura } \\
\text { militar } \\
\text { 1976-1983 }\end{array}$} \\
\hline 23 & Ricardo P. Bruera & 29 de marzo de 1976 - 28 de mayo de 1977 & \\
\hline 24 & Juan José Catalán & 25 de junio de 1977 - 26 de agosto de 1978 & \\
\hline 25 & $\begin{array}{l}\text { Juan Rafael } \\
\text { Llerena Amadeo }\end{array}$ & 5 de noviembre de 1978 - 29 de marzo de 1981 & \\
\hline 26 & $\begin{array}{l}\text { Carlos } \\
\text { Burundarena }\end{array}$ & 29 de marzo de 1981 - 12 de diciembre de 1981 & \\
\hline 27 & $\begin{array}{l}\text { Cayetano } \\
\text { Licciardo }\end{array}$ & 22 de diciembre de 1981 -10 de diciembre de 1983 & \\
\hline 28 & $\begin{array}{l}\text { Carlos Alconada } \\
\text { Aramburú }\end{array}$ & 10 de diciembre de 1983 - 21 de junio de 1986 & \multirow{4}{*}{ Alfonsin } \\
\hline 29 & Julio Rajneri & 21 de junio de 1986 - 10 de septiembre de 1987 & \\
\hline 30 & $\begin{array}{l}\text { Jorge Federico } \\
\text { Sábato }\end{array}$ & 10 de septiembre de 1987 - 26 de mayo de 1989 & \\
\hline 31 & $\begin{array}{l}\text { José Gabriel } \\
\text { Dumón }\end{array}$ & 26 de mayo de 1989 - 8 de julio de 1989 & \\
\hline 32 & Antonio Salonia & 8 de julio de 1989 - 4 de diciembre de 1992 & \multirow{4}{*}{ Menem 1} \\
\hline 33 & $\begin{array}{l}\text { Jorge Alberto } \\
\text { Rodríguez }\end{array}$ & 4 de diciembre de 1992 - 28 de marzo de 1996 & \\
\hline 34 & Susana Decibe & 28 de marzo de 1996 - 7 de mayo de 1999 & \\
\hline 35 & $\begin{array}{l}\text { Manuel } \\
\text { García Solá }\end{array}$ & 7 de mayo de 1999 - 10 de diciembre de 1999 & \\
\hline
\end{tabular}




\begin{tabular}{|c|c|c|c|}
\hline & $\begin{array}{l}\text { Ministro de } \\
\text { educación }\end{array}$ & Periodo & Presidencia \\
\hline 36 & Juan José Llach & 10 de diciembre de 1999 - 25 de septiembre de 2000 & \multirow{3}{*}{ De la Rúa } \\
\hline 37 & Hugo Juri & 25 de septiembre de 2000 - 20 de marzo de 2001 & \\
\hline 38 & Andrés Delich & 20 de marzo de 2001 - 21 de diciembre de 2001 & \\
\hline 39 & $\begin{array}{l}\text { Graciela } \\
\text { Giannettasio }\end{array}$ & 3 de enero de 2002 - 25 de mayo de 2003 & Duhalde \\
\hline 40 & Daniel Filmus & 25 de mayo de 2003 - 10 de diciembre de 2007 & Kirchner \\
\hline 41 & $\begin{array}{l}\text { Juan Carlos } \\
\text { Tedesco }\end{array}$ & 10 de diciembre de 2007 - 20 de julio de 2009 & \multirow{2}{*}{$\begin{array}{l}\text { Fernández de } \\
\text { Kirchner } 1 \\
\text { Fernández de } \\
\text { Kirchner } 2\end{array}$} \\
\hline 42 & Alberto Sileoni & 20 de julio de 2009 - 10 de diciembre de 2015 & \\
\hline 43 & Esteban Bullrich & 17 de julio de 2017 - 5 de septiembre de 2018 & \multirow[b]{2}{*}{ Macri } \\
\hline 44 & $\begin{array}{l}\text { Alejandro } \\
\text { Finocchiaro }\end{array}$ & 5 de septiembre de 2018-10 de diciembre de 2019 & \\
\hline 45 & Nicolás Trotta & 10 de diciembre de 2019 - en el cargo & Fernández \\
\hline
\end{tabular}

Fuente: elaboración propia.

Anexo 2. Materias escolares destinadas a la educación política según periodos y contextos

\begin{tabular}{|c|c|c|}
\hline Periodo & Asignatura & Características del periodo \\
\hline 1952-1955 & $\begin{array}{l}\text { Cultura } \\
\text { ciudadana }\end{array}$ & $\begin{array}{l}\text { - Se atienden demandas sociales } \\
\text { controladas desde un Estado social. } \\
\text { Movimientismo. Partido y sindicatos } \\
\text { imbricados en la estructura estatal. } \\
\text { Reconocimiento amplio de derechos } \\
\text { sociales y económicos. } \\
\text { Sistema internacional bipolar. }\end{array}$ \\
\hline 1956-1972 & $\begin{array}{l}\text { Educación } \\
\text { democrática }\end{array}$ & $\begin{array}{l}\text { - } \quad \text { Se recortan las demandas vía } \\
\text { intervención de los sindicatos. } \\
\text { - } \quad \text { Estado liberal - benefactor / Estado } \\
\text { burocrático autoritario. } \\
\text { - } \quad \text { Gobiernos de facto. } \\
\text { - } \quad \text { Proscripción del partido mayoritario. } \\
\text { - } \quad \text { Intervención de los sindicatos. } \\
\text { - } \quad \text { Represión. } \\
\text { Reconocimiento de derechos sociales y económicos } \\
\text { - Art. } 14 \text { bis), recorte de los derechos políticos. } \\
\text { Guerra Fría. }\end{array}$ \\
\hline
\end{tabular}




\begin{tabular}{|c|c|c|}
\hline Periodo & Asignatura & Características del periodo \\
\hline 1973-1976 & ERSA & $\begin{array}{ll} & \text { Estado incapaz de atender las demandas. } \\
\text { - } & \text { Crisis económica. } \\
\text { Situación de "empate" que impide } \\
\text { efectivizar la dominación estatal. } \\
\text { - } \quad \text { Libre funcionamiento del sistema } \\
\text { partidario (bipartidismo). } \\
\text { Escisión del peronismo, exclusión política } \\
\text { de la izquierda. Guerrilla y represión. } \\
\text { Movimiento de países del Tercer Mundo. }\end{array}$ \\
\hline 1976-1983 & $\begin{array}{l}\text { Formación } \\
\text { cívica- } \\
\text { Formación } \\
\text { moral y } \\
\text { cívica } \\
\text { Dictadura }\end{array}$ & $\begin{array}{ll}\text { - } & \text { Estado terrorista. } \\
\text { - } & \text { Represión planificada y sistemática. } \\
\text { - } & \text { Supresión de los partidos políticos. } \\
\text { - } & \text { Eliminación de los sindicatos. } \\
\text { - } & \text { Censura. } \\
\text { - } & \text { Exclusión política y social. } \\
\text { - } & \text { Se inicia el proceso de recorte de los } \\
& \text { derechos sociales y económicos. } \\
\text { - } & \text { Agotamiento del Estado benefactor. }\end{array}$ \\
\hline $\begin{array}{l}1983- \\
1992\end{array}$ & $\begin{array}{l}\text { Educación } \\
\text { cívica }\end{array}$ & $\begin{array}{l}\text { - } \quad \text { Crisis económica. Hiperinflación. } \\
\text { - } \quad \text { Incapacidad estatal para atender las demandas. } \\
\text { - } \quad \text { Aibre juego electoral, partidario, sindical, etcétera. } \\
\text { - } \quad \text { Reconocimiento amplio de derechos. } \\
\text { - Imposibilidad de incluir totalmente a } \\
\text { causa de la crisis económica. }\end{array}$ \\
\hline 1993-2003 & $\begin{array}{l}\text { Formación } \\
\text { ética y } \\
\text { ciudadana }\end{array}$ & 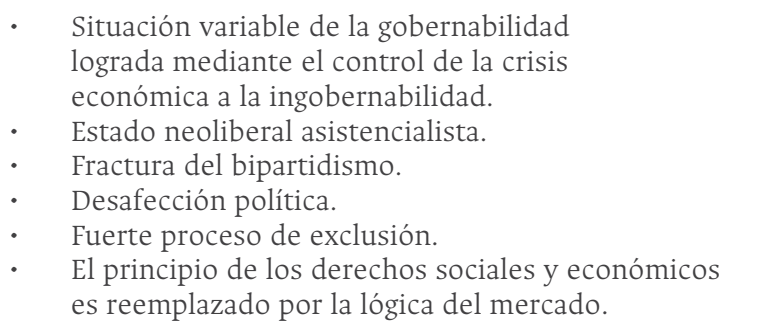 \\
\hline 2007 & $\begin{array}{l}\text { Construcción } \\
\text { de la } \\
\text { ciudadanía }\end{array}$ & $\begin{array}{ll}\text { - } & \text { Estado social post crisis. } \\
\text { - } & \text { Estado garante de derechos socio económicos. } \\
\text { - } & \text { Desaricas socio económicas distributivas. } \\
\text { - } & \text { Legitimidad política. } \\
\text { - } & \text { Paradigma inclusivo. } \\
\text { - } & \text { Enfoque de derechos humanos y } \\
\text { - } & \text { profundización democrática. } \\
\text { - } & \text { Políticas públicas integrales para la inclusión social. } \\
& \text { Parión de la sociedad civil. }\end{array}$ \\
\hline
\end{tabular}




\begin{tabular}{|c|c|c|}
\hline Periodo & Asignatura & Características del periodo \\
\hline 2010 & $\begin{array}{l}\text { Salud y } \\
\text { adolescencia }\end{array}$ & $\begin{array}{ll}\text { - } & \text { Estado social post crisis. } \\
\text { - } & \text { Estado garante de derechos socio económicos. } \\
\text { - } & \text { Desarricas socio económicas distributivas. } \\
& \text { recuperación del empleo interno y } \\
\text { - } & \text { Legitimidad política. } \\
\text { - } & \text { Paradigma inclusivo. } \\
\text { - } & \text { Enfoque de derechos humanos y } \\
\text { - } & \text { Profundización democrática. } \\
\text { - } & \text { Particas públicas integrales para la inclusión de la social } \\
& \text { Podad civil. }\end{array}$ \\
\hline 2011 & $\begin{array}{l}\text { Política y } \\
\text { ciudadanía }\end{array}$ & $\begin{array}{ll}\text { - } & \text { Estado social post crisis. } \\
\text { - } & \text { Estado garante de derechos socio económicos. } \\
\text { - } & \text { Desaricas socio económicas distributivas. } \\
& \text { recuperación del emico interno y } \\
\text { - } & \text { Legitimidad política. } \\
\text { - } \quad \text { Paradigma inclusivo. } \\
\text { Enfoque de derechos humanos y } \\
\text { profundización democrática. } \\
\text { - Políticas públicas integrales para la inclusión social } \\
\quad \text { Participación de la sociedad civil. }\end{array}$ \\
\hline 2011 & $\begin{array}{l}\text { Trabajo y } \\
\text { ciudadanía }\end{array}$ & $\begin{array}{ll}\text { - } & \text { Estado social post crisis. } \\
\text { - } & \text { Estado garante de derechos socio económicos. } \\
\text { - } & \text { Políticas socio económicas distributivas. } \\
& \text { } \text { recuperrocilo económico interno y } \\
\text { - } & \text { Legitimidad política. } \\
\text { - } & \text { Paradigma inclusivo. } \\
\text { - } & \text { Enfoque de derechos humanos y } \\
& \text { profundización democrática. } \\
\text { - } & \text { Políticas públicas integrales para la inclusión social } \\
\text { - } & \text { Participación de la sociedad civil. }\end{array}$ \\
\hline
\end{tabular}

Fuente: elaboración propia. 